\title{
Alginate coated chitosan nanoparticles are an effective subcutaneous adjuvant for hepatitis B surface antigen
}

\author{
Olga Borges ${ }^{\mathrm{a}, *}$, Marta Silva ${ }^{\mathrm{b}}$, Adriano de Sousa ${ }^{\mathrm{a}}$, Gerrit Borchard ${ }^{\mathrm{c}}$, \\ Hans E. Junginger ${ }^{\mathrm{d}}$, Anabela Cordeiro-da-Silva ${ }^{\mathrm{b}}$ \\ a Center for Pharmaceutical Studies, Faculty of Pharmacy, University of Coimbra, 3000-295 Coimbra, Portugal \\ b IBMC-Instituto de Biologia Molecular e Celular, Universidade do Porto, 4050-047 Porto, Portugal \\ c School of Pharmaceutical Sciences Geneva-Lausanne, University of Geneva, 1211 Geneva 4, Switzerland \\ ${ }^{\mathrm{d}}$ Faculty of Pharmaceutical Sciences, Naresuan University, Phitsanulok 65 000, Thailand
}

\section{A R T I C L E I N F O}

\section{Article history:}

Received 18 March 2008

Received in revised form 10 June 2008

Accepted 19 August 2008

\section{Keywords:}

Adjuvants

Subcutaneous vaccination

Hepatitis B surface antigen

CpG oligodeoxynucleotide

Alginate coated chitosan nanoparticles

\begin{abstract}
A B S T R A C T
We recently described a delivery system that is composed of a chitosan core to which the hepatitis B surface antigen (HBsAg) was adsorbed and subsequently coated with sodium alginate. In this present work, alginate coated chitosan nanoparticles were evaluated as a subcutaneous adjuvant for HBsAg.

HBsAg loaded, alginate coated or uncoated chitosan nanoparticles, associated or not with CpGODN were subcutaneously administered to mice and several immunological parameters were evaluated.

A high anti-HBsAg IgG titer $(2271 \pm 120 \mathrm{mIU} / \mathrm{ml})$, with the majority of antibodies being of Th2 type, was observed within group I, vaccinated with HBsAg loaded onto coated nanoparticles. However, regarding cellular immune response, no significant differences were observed for antigen-specific splenocyte proliferation or for the secretion of IFN- $\gamma$ and IL-4, when compared to the control group. The co-delivery of antigen-loaded nanoparticles in the presence of the immunopotentiator, CpG ODN 1826, resulted in an increase of anti-HBsAg IgG titers that was not statistically different from the first group; however, an increase of the IgG2a/IgG1 ratio from 0.1 to 1.0 and an increase $(p<0.01)$ of the IFN- $\gamma$ production by the splenocytes stimulated with the HBV antigen was observed.

The enhancement of the immune response observed with the antigen-loaded nanoparticles demonstrated that chitosan is a promising platform for parenteral HBsAg delivery and, when co-administered with the CpG ODN, resulted in a mixed Th1/Th2 type immune response.
\end{abstract}

(C) 2008 Elsevier B.V. All rights reserved.

\section{Introduction}

Chitosan, a biodegradable and biocompatible polysaccharide with immunological activity [1,2], which acts both as a bioadhesive [3] and as efficient absorption enhancer [4], has also been regarded as a promising polymer for the formulation of vaccine delivery systems, especially for application to mucosal surfaces [5]. Recently, we have designed a delivery system composed of a chitosan core to which the hepatitis B surface antigen (HBsAg) was adsorbed and was then coated with sodium alginate. This delivery system was recently evaluated for mucosal routes of immunization.

On the other hand, the evaluation of chitosan as an adjuvant for parenteral vaccination has been less studied and, in most cases, the results of these vaccination studies were reported together with the results of intranasal or oral vaccination studies, making the possible value of

\footnotetext{
* Corresponding author. Center for Pharmaceutical Studies, Faculty of Pharmacy, University of Coimbra, Rua do Norte, 3000-295 Coimbra, Portugal. Tel.: +351 239859927; fax: +351 239827126

E-mail address: olga@ci.uc.pt (0. Borges).
}

chitosan as an adjuvant for parenteral routes less noticeable in the scientific literature. Generally speaking, the development of safe novel adjuvants is necessary not only for the more challenging environment of the mucosal surfaces, but also for parenteral vaccination, to maximize the efficacy of new or already available vaccines. In the last few years this idea became even more urgent since newer generations of antigens are predominantly purified recombinant proteins, which are often poorly immunogenic. Additionally, the new generation of adjuvants may also allow vaccination strategies to be applied to novel areas, including "therapeutic" vaccines designed to control allergies, auto-immune diseases, malignancies, drug dependencies, neural diseases, or fertility [6].

Despite the efficacy of hepatitis B vaccines, immunization failure may occur and can sometimes be explained by several factors such as improper storage or administration of the vaccines, advanced age of the individual, chronic liver disease, and immunosuppression. Genetically determined resistance appears to be another important factor causing a non-responder rate of up to $10 \%[7,8]$. Moreover, all the conventional licensed hepatitis B vaccines in use contain alum as adjuvant. Although it is a potent B cell stimulator, alum is less effective in inducing a Th1 response by the intramuscular route [9]. The Th1 type immune responses, 
characterized by secretion of interferon- $\gamma$ (IFN- $\gamma$ ), tumour necrosis factor- $\alpha$ (TNF- $\alpha$ ), and opsonizing antibodies such as the IgG2a isotype, as well as strong cytotoxic T-lymphocyte (CTL) induction, are necessary for the control of intracellular infections, such as viral infections [10-12]. In contrast, the development of a strong Th2 response, which is characterized by the secretion of IL- 4 and IL- 5 cytokines and antibodies such as IgG1 and IgE, is more useful in combating extracellular infections [12].

The viral clearance in acute, self-limited hepatitis B virus infection correlates with increasing CTL and T-helper cell activities and the occurrence of anti-HBs antibodies [13]. In contrast, patients suffering from chronic hepatitis B have insufficient or absent immune responses $[9,13]$. On the other hand, experimental data support the hypothesis that the enhancement of HBV-specific immune reactions could have some beneficial effects in the therapy of chronic hepatitis B [13]. Therefore, it has been suggested [9] that the administration of the hepatitis B vaccine alone, or in combination with diverse cytokines, should be evaluated in future studies for treatment of chronic hepatitis B virus infection. The usefulness of this therapeutic vaccination may be further improved if the vaccine was able to induce a stronger Th1 immune response.

The differentiation of an antigen specific CD4 T-helper subset (Th1 or Th2) takes place at the time of priming, and the type of stimulated CD4 subset will depend on a number of factors, including the cytokine environment [10]. The adjuvant used can alter the cytokine environment at the site of the primary immune response [10]. Therefore, the selection of an appropriate adjuvant is the first step toward the successful induction of an appropriate immune response.

In the present study, the above mentioned alginate coated chitosan nanoparticles were investigated for the first time as adjuvant for subcutaneous vaccination with the recombinant hepatitis B surface antigen. Moreover, this paper investigates the co-administration of CpG ODN 1826, a potent adjuvant in mice that was shown to induce Th1 type immune response in combination with a number of different antigens, such as influenza virus [14], hepatitis B antigen [15-17], and tetanus toxoid [18], with the nanoparticles.

\section{Materials and methods}

\subsection{Materials}

\subsubsection{Polymers}

Chitosan was purchased from Primex BioChemicals AS (Avaldsnes, Norway). According to the provider's specifications, the degree of deacetylation was $95 \%$ (titration method) and the viscosity $8 \mathrm{cP}$ (measured in $1 \%$ solutions in $1 \%$ acetic acid). A low molecular weight pharmaceutical grade sodium alginate (MANUCOL LB ${ }^{\circledR}$ ) was kindly donated by ISP Technologies Inc. (Surrey, UK). According to the provider's specifications, the typical values for the percentage of mannuronic and guluronic acid for Manucol LB were 61\% and 39\%, respectively, with an estimated molecular weight of $18 \mathrm{kDa}$.

\subsubsection{Antigen, adjuvant and reagents}

The hepatitis B surface antigen (HBsAg), (subtype ADW2) was kindly provided by GSK Biologicals (Rixensart, Belgium), Class B, CpG ODN (1826) (5'-TCC ATG ACG TTC CTG ACG TT-3') was purchased from Coley Pharmaceutical Group (Ottawa, Canada).

Concanavalin A (Con A), phenylmethanesulfonyl fluoride (PMSF), avidin peroxidase conjugate and the BCIP/NBT-purple liquid substrate system for membranes were purchased from Sigma Chemicals (St. Louis, USA). Certified foetal bovine serum (FBS) and L-glutamine (200 mM) were from Gibco (Invitrogen Co, Paisley, Scotland, UK), 1 M HEPES buffer $(0.85 \%$ $\mathrm{NaCl}$ ), RPMI 1640 without L-glutamine and Pen-Strep (10,000 U penicillin/ $\mathrm{ml} ; 10,000 \mu \mathrm{g}$ streptomycin/ml) were from Biowhitaker (Cambrex Bio Science, Verviers, Belgium). [methyl- ${ }^{3} \mathrm{H}$ ] thymidine $(1.0 \mathrm{mCi} / \mathrm{ml})$ was obtained from Amersham Biosciences (UK) and R-phycoerythrin (PE)conjugated hamster anti-mouse CD69, fluorescein isothiocyanate (FITC)- conjugated rat anti-mouse CD4 and FITC-conjugated rat anti-mouse CD8 were obtained from BD Biosciences (Madrid, Spain). The FITC-conjugated goat anti-mouse IgM (anti- $\mu$ ), the anti-mouse IFN- $\gamma$ and biotin rat antimouse IFN- $\gamma$ was purchased from PharMingen (San Diego, CA, USA). The mouse IgA ELISA quantification kit was obtained from Bethyl Laboratories, (Montgomery, USA). All other reagents used were of analytical grade. All solutions were prepared in ultrapure water.

\subsection{Methods}

\subsubsection{Preparation of the coated nanoparticles}

The preparation of the alginate coated chitosan nanoparticles was performed according to the method previously described [19]. In brief, chitosan was dissolved at a concentration of $0.25 \%(\mathrm{w} / \mathrm{v})$ in diluted acetic acid solution. The formation of the particles was achieved by the addition of $3.5 \mathrm{ml}$ of sodium sulphate solution $(10 \% \mathrm{w} / \mathrm{v})$ to $200 \mathrm{ml}$ of the chitosan solution. The resulting suspension was centrifuged for $30 \mathrm{~min}$ at $3500 \mathrm{rpm}(2800 \times \mathrm{g})$ and the supernatant was discarded. The particles were re-suspended in Millipore water and centrifuged twice. They were finally frozen in liquid nitrogen and freeze-dried overnight using a Labconco freeze dry system (Labconco Corporation, Kansas, USA). The dry powder was kept frozen until further use.

The loading of the chitosan nanoparticles with HBsAg was performed by incubating a solution of HBsAg with a suspension of chitosan particles in phosphate buffer of $\mathrm{pH} 7.4$ under mild agitation at room temperature for $120 \mathrm{~min}$. The resulting suspension composed of $0.015 \%(\mathrm{w} / \mathrm{v}) \mathrm{HBsAg}$ and $0.5 \%(\mathrm{w} / \mathrm{v})$ nanoparticles was used in the subsequent coating step. Alginate coated nanoparticles were obtained by mixing equal volumes of the HBsAg loaded nanoparticle suspension and a solution of sodium alginate in phosphate buffer $(1 \% \mathrm{w} / \mathrm{v})$ under magnetic stirring. The agitation was maintained for $20 \mathrm{~min}$. The suspension was then centrifuged for $10 \mathrm{~min}$ at $1600 \mathrm{rpm}$ and the supernatant was discarded. The particles were re-suspended in $0.262 \mathrm{mM} \mathrm{CaCl}_{2}$ in $50 \mathrm{mM}$ HEPES buffer solution, kept under agitation for another $10 \mathrm{~min}$, and immediately administered to mice group I. Group II received the same 1\% particle suspension to which CpG ODN had previously been added. Finally, a $1 \%$ chitosan nanoparticle suspension, which contained $\mathrm{CpG}$ and the antigen, but was not coated with alginate, was administered to mouse group III.

\subsubsection{Evaluation of the loading efficacy of $\mathrm{HBs} A g$ and $C p G$ ODN in coated and uncoated nanoparticles}

The loading efficacy of the coated and uncoated nanoparticles was calculated indirectly by quantifying the antigen or the CpG ODN that remained in solution as previously described $[20,21]$.

The loading efficacy (LE) and the loading capacity (LC) were calculated from the following equations:

$$
\begin{aligned}
\mathrm{LE}(\%)= & (\text { Total amount of HBsAg-free HBsAg }) \\
& / \text { Total amount of HBsAg*100 }
\end{aligned}
$$

$$
\begin{aligned}
& \mathrm{LC}(\mu \mathrm{g} \text { of } \mathrm{HBsAg} / \mathrm{mg} \text { nanoparticles dry weight }) \\
& =(\text { Total amount of HBsAg-free } \mathrm{HBsAg}) \\
& \text { /mg chitosan nanoparticles dry weight. }
\end{aligned}
$$

\subsubsection{Immunization studies}

2.2.3.1. Animals. Seven week-old female BALB/cAnNHsd mice were used (Harlan Iberica, Barcelona, Spain) with four mice per group. Animals were housed for acclimatization one week before the experiments at the animal resource facilities of the Faculty. Animal care, handling, and immunization protocols were adhered to the "Principles of Laboratory Animal Care" and in accordance with institutional ethical guidelines. The mice had free access to food and water and were kept under a $12 \mathrm{~h}$ light/dark cycle. 
Table 1

Resume of the formulations s.c. administered to mice groups

\begin{tabular}{lllll}
\hline & Group I & Group II & Group III & Group IV \\
\hline Chitosan nanoparticles (NP) & $\mathrm{X}$ & $\mathrm{X}$ & $\mathrm{X}$ & \\
Alginate coating & $\mathrm{X}$ & $\mathrm{X}$ & & \\
$10 \mu \mathrm{g}$ HBsAg loaded on NP & $\mathrm{X}$ & $\mathrm{X}$ & $\mathrm{X}$ & $\mathrm{X}$ \\
$10 \mu \mathrm{g}$ HBsAg in PBS solution & & & & \\
$20 \mu \mathrm{g}$ CpGODN loaded on NP & & & $\mathrm{X}$ & \\
$20 \mu \mathrm{g}$ CpG ODN in solution & & $\mathrm{X}$ & & \\
\hline
\end{tabular}

Each mouse received a volume of $100 \mu \mathrm{g}$ of the formulation.

2.2.3.2. Treatment groups (see also Table 1). Group I- suspension of alginate coated chitosan nanoparticles loaded with $10 \mu \mathrm{g}$ HBsAg.

Group II- suspension of alginate coated chitosan nanoparticles loaded with $10 \mu \mathrm{g}$ HBsAg plus $20 \mu \mathrm{g}$ of CpG ODN in solution.

Group III- suspension of the chitosan nanoparticles loaded with $10 \mu \mathrm{g}$ HBsAg and $20 \mu \mathrm{g}$ CpG ODN.

Group IV- Phosphate buffer saline (PBS; pH 7.4) solution with $10 \mu \mathrm{g}$ $\mathrm{HBsAg}$ (reference group).

Group V- untreated or control.

2.2.3.3. Immunization schedule. The primary immunization was followed by one boost with three weeks between immunizations. The mice were sacrificed four weeks after the boost. To evoke an immune response, a total volume of $100 \mu$ of the formulations was subcutaneously administered to non-anesthetized mice.

2.2.3.4. Collection of samples. Blood samples were taken from the orbital sinus before the boost and by cardiac puncture at the end of the experiment. The sera were prepared by centrifugation and stored at $-20{ }^{\circ} \mathrm{C}$ until analysis.

Faecal pellets (4-8) were collected in Eppendorf tubes four and one days before the end of the experiment. The pellets $(0.2 \mathrm{~g} / \mathrm{ml})$ were suspended in PBS (containing 0.1\% sodium azide, 0.1\% BSA, and $1 \mathrm{mM}$ PMSF), vortexed, and allowed to rest at room temperature for $1 \mathrm{~h}$. Solid matter was separated by centrifugation at $14,000 \mathrm{rpm}$ for $15 \mathrm{~min}$. The clear supernatants were frozen at $-80^{\circ} \mathrm{C}$ until determination of antigenspecific and total secretory IgA by ELISA.

2.2.3.5. Enzyme-linked immunosorbent assays (ELISA) for HBsAg specific immunoglobulins. Ninety-six-well flat-bottomed microtiter plates (Nunc immunoplate maxisorb) were previously coated with recombinant HBsAg ( $1 \mu \mathrm{g} /$ well) dissolved in coating buffer (50 mM sodium carbonate, $\mathrm{pH} 9.6$ ) by overnight incubation at $4{ }^{\circ} \mathrm{C}$. The plates were washed five times with PBS-T (PBS containing 0.05\% Tween-20) and blocked with $3 \%$ BSA in PBS-T ( $200 \mu \mathrm{l} /$ well) for $1 \mathrm{~h}$ at $37^{\circ} \mathrm{C}$. The plates were then washed five times with PBS-T and the serial dilutions of each serum (100 $\mu \mathrm{l} /$ well) from the individual mice were tested in triplicate, starting from a 1:100 dilution in PBS-T. The serum was incubated for $2 \mathrm{~h}$ at $37^{\circ} \mathrm{C}$ and after washing the plates with PBS-T, the plates were incubated for additional $30 \mathrm{~min}$ at $37^{\circ} \mathrm{C}$ with peroxidaselabelled goat anti-mouse immunoglobulin $\mathrm{G}$ and isotypes (anti-IgG1, anti-IgG2a, anti-IgG2b, and anti-IgG3). The bound antibodies were revealed by adding $100 \mu \mathrm{l} /$ well of $0.5 \mathrm{mg} / \mathrm{ml}$ of 0 -phenylenediamine dihydrochloride (OPD) (Sigma, Spain) in $10 \mathrm{ml}$ of citrate buffer with $10 \mu \mathrm{l}$ of $\mathrm{H}_{2} \mathrm{O}_{2}$. The reaction was stopped after $10 \mathrm{~min}$ with $50 \mu \mathrm{l}$ of $3 \mathrm{M}$ $\mathrm{HCl}$ to each well. The absorbance was read at $492 \mathrm{~nm}$ in an automatic ELISA reader (Easy Reader 400, SLT-LABINSTRUMENTS). ELISA titers were expressed as $\mathrm{mIU} / \mathrm{ml}$, and $1 \mathrm{mIU}$ is the mean OD of the preimmune serum plus two times the standard deviation.

The measurement of IgA was carried out using a mouse IgA ELISA quantification kit (Bethyl Laboratories, Montgomery, Texas, USA) as described by the manufacturer. In order to measure the sIgA levels in the faeces, total slgA and the specific anti-HBs slgA were determined in the extracts. The results are presented as the anti-HBsAg IgA/total
IgA. By this way, variations between samples related with the extraction process or stability of the sIgA were minimized.

The IgA standard was diluted to appropriate concentrations in PBS with $1 \%$ BSA to create a calibration curve. The gut washes were diluted in PBS-T with 1\% BSA and added to the plates in series of two-fold dilutions. The concentrations of the total and specific IgA were determined from the calibration curve generated for each set of samples using a four parameter logistic curve-fit generated by SigmaPlot software (version 8.0, SPSS Inc).

2.2.3.6. Preparation of spleen cell suspensions. The mice were euthanized by cervical dislocation and their spleens were aseptically removed. Individual spleen cell suspensions were prepared in a Petri dish using curved needles and washed twice with RPMI 1640. The final suspension was adjusted to a final concentration of $1 \times 10^{7}$ cells/ $\mathrm{ml}$ in complete RPMI 1640 medium supplemented with $10 \%(\mathrm{v} / \mathrm{v})$ foetal bovine serum (FBS), 1\% (v/v) glutamine, 1\% (v/v) Pen-Strep, and $2 \%(\mathrm{v} / \mathrm{v}) 1 \mathrm{M}$ HEPES buffer.

2.2.3.7. Splenocyte cell culture in the presence of the mitogens. Using sterile 96-well flat-bottomed tissue culture plates, $25 \mu$ of splenocyte suspension $\left(1 \times 10^{7}\right.$ cells/ ml) from each mouse were plated in triplicate with $25 \mu \mathrm{l}$ of concentrated RPMI solution of the mitogen [Con A $(50 \mu \mathrm{g} / \mathrm{ml})$, CpG ODN $(50 \mu \mathrm{g} / \mathrm{ml})$ plus HBsAg ( $16 \mu \mathrm{g} / \mathrm{ml}), \mathrm{HBsAg}(16 \mu \mathrm{g} / \mathrm{ml})$ alone, or without mitogen (control)]. Finally, the volume of the well was filled up to $200 \mu \mathrm{l}$ with complete RPMI and incubated according to the following conditions (see Sections 2.2.3.8 and 2.2.3.9).

2.2.3.8. Cytokine production by splenocytes. Spleen cell suspensions were plated with the appropriate mitogen (see Section 2.2.3.7) and incubated in a humidified $5 \% \mathrm{CO}_{2}$ incubator for $48 \mathrm{~h}$ (IL-4, IL-10) and $96 \mathrm{~h}($ IFN- $\gamma)$ at $37{ }^{\circ} \mathrm{C}$. The plates were centrifuged and the clear supernatants stored at $-80^{\circ} \mathrm{C}$ until analysis of the cytokines by ELISA technique described elsewhere [22].

2.2.3.9. Lymphoproliferation assay. Splenocytes were obtained and cultured together with the mitogens in flat-bottomed 96-well plates as described before (see Section 2.2.3.7). The cells were cultured for $96 \mathrm{~h}$ at $37^{\circ} \mathrm{C}$ and during the last $8 \mathrm{~h}$ of incubation, each well was pulsed with $1 \mu \mathrm{Ci}$ of [methyl ${ }^{3} \mathrm{H}$ ] thymidine. The 96 well plates with the cells were stored at $-20{ }^{\circ} \mathrm{C}$ until further analysis. The cells were later thawed and harvested onto a fiberglass filter (filter mats, molecular devices, Skatron, Lier, Norway) using a semiautomatic cell harvester (Scatron Instruments, USA) and DNA thymidine incorporation was counted by standard liquid scintillation techniques with a Beckman LS 6500 scintillation counter (Beckman Coulter Inc., Fullerton, USA). Thymidine incorporation was expressed as counts per minute (cpm).

\subsection{Statistical analysis}

If another method is not explicitly stated, the data are presented as the mean \pm S.E.M. for at least three experiments and statistical significance was assessed using one-way analysis of variance (ANOVA) followed by Dunnett's post test for comparing the vaccinated groups with the control group. The Kruskal-Wallis test, followed by Dunn's multiple comparison test, were used for comparing the cytokine values between the groups using the Prism 4 (GraphPad software, CA, USA). Differences were considered significant when $p<0.05$.

\section{Results}

\subsection{Characterization of the alginate coated chitosan nanoparticles}

Our group recently reported on the development of alginate coated chitosan nanoparticles and the characterization of this new delivery system [19]. Briefly, before coating with sodium alginate, chitosan 


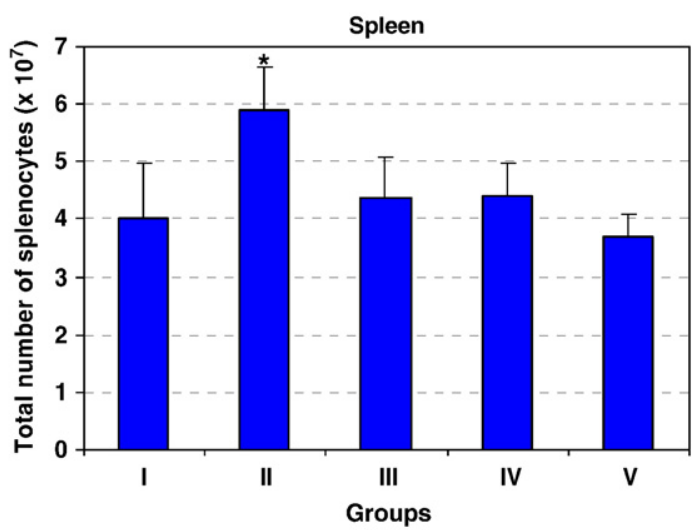

Fig. 1. Total number of splenocytes from groups (I, II, III, IV and V). Each bar corresponds to the group geometric mean plus the standard error of the mean. $\left({ }^{*} p<0.05 ; t\right.$-test).

nanoparticles were positively charged $(+37 \mathrm{mV} \pm 3.6)$ and after the coating with alginate they became negatively charged $(-34.9 \mathrm{mV} \pm$ 8.3). Chitosan nanoparticles had a mean diameter of $643 \pm 171.7 \mathrm{~nm}$ measured by dynamic light scattering technique. More recently [20], we published the results of ovalbumin (model vaccine) release studies from coated and uncoated nanoparticles. Ovalbumin release studies, performed in several buffers at different $\mathrm{pH}$ values, allowed us to conclude that the coating of the ovalbumin loaded chitosan nanoparticles prevented an ovalbumin burst release which was observed with the uncoated chitosan nanoparticles at $\mathrm{pH} 5.5 ; 6.8$, and 7.4 (phosphate buffer) within the first $30 \mathrm{~min}$ of incubation.

\subsection{Hepatitis $B$ antigen and $C p G$ adsorption to nanoparticles}

Hepatitis B antigen was efficiently associated with alginate coated chitosan nanoparticles. The loading efficacy of hepatitis B vaccine in the coated nanoparticles was $77.1 \pm 3.0 \%$ (mean \pm STDEV) and the mean of the loading capacity was $23.1 \mu \mathrm{g}$ of $\mathrm{HBsAg} / \mathrm{mg}$ of dry chitosan nanoparticles \pm 2.1 (STDEV). The adsorption efficacy of CpG to the chitosan nanoparticles was $97.0 \pm 1.3 \%$ and the loading capacity was $29.0 \pm 0.03$ ( $\mu \mathrm{g}$ of CPG ODN/mg of dry chitosan nanoparticles).

3.3. Cellular immune response to subcutaneous administration of $\mathrm{HBs} \mathrm{Ag}$ associated with the chitosan nanoparticles

The total number of splenocytes was evaluated on week four after the boost and the results are shown in Fig. 1. At this time point the group II showed a higher number of cells in the spleen when compared with control group (Group V). Additionally, possible differences in the maturation state of the splenocytes were also studied. Two of the parameters observed were their ability to proliferate (Fig. 2) and to produce cytokines (Fig. 3) after in vitro stimulation with $\mathrm{HBsAg}$, HBsAg+ CpGODN, and Con A (positive control). As expected, after $96 \mathrm{~h}$ of culture without any external stimulation (Fig. 2A), the splenocytes did not exhibit significant proliferative activity. Group IV was an exception, with a mean value statistically higher $(p<0.05)$ than the control group; however with a high variability within the group (Fig. 2A). The same behaviour was observed when the splenocytes from the same group IV were in vitro stimulated with the antigen (Fig. $2 \mathrm{~B}$ ) or with the antigen + CpG ODN (Fig. 2C), but not with Con A (Fig. 2D). A Similar cellular

Fig. 2. Lymphoproliferative response after sc administration of the different hepatitis $B$ vaccine formulations. In vitro proliferation of individual mouse splenocytes for a $96 \mathrm{~h}$ period stimulated with: A)-without stimulus. B)-HBsAg. C)-HBsAg + CpG ODN. D)Con A. Each circle represents the result of individual samples and the horizontal bar the mean of the group. The results corresponded to the thymidine incorporation and are expressed as counts per minute (cpm). immune response was observed within the group II, where a higher proliferative response was selective to the presence of the antigen alone $(p<0.01)$ (Fig. 2B) or the antigen plus the CpG ODN $(p<0.05)$ (Fig. 2C).

A A Lymphoproliferative assay

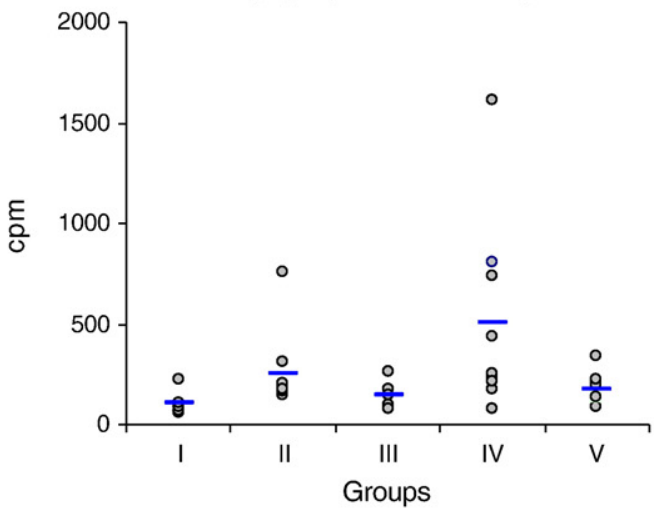

B HBsAg

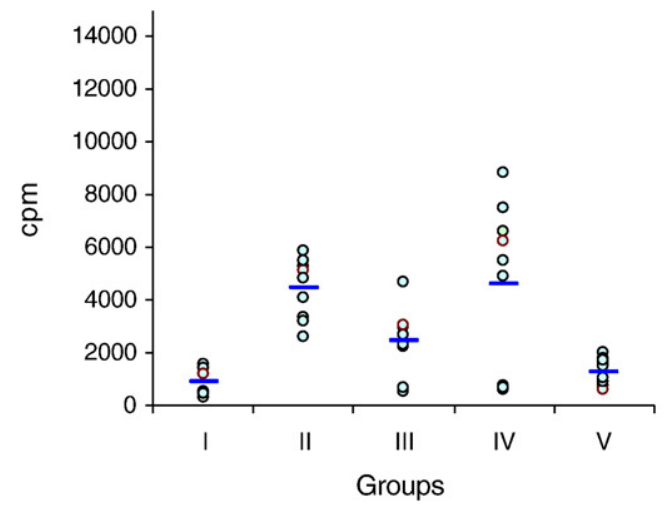

C HBsAg + CpG ODN

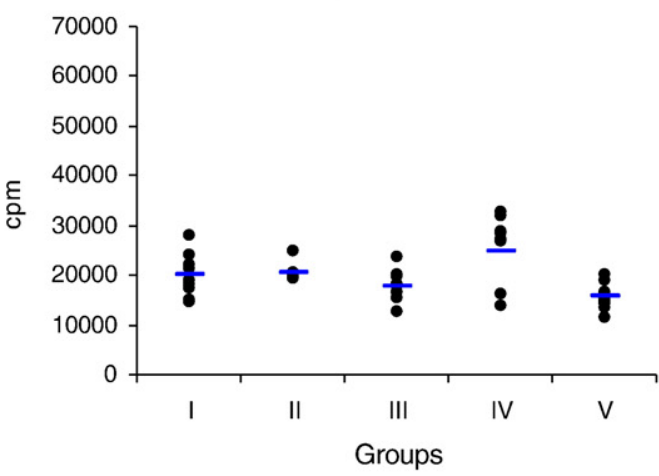

D $\operatorname{con} \mathrm{A}$

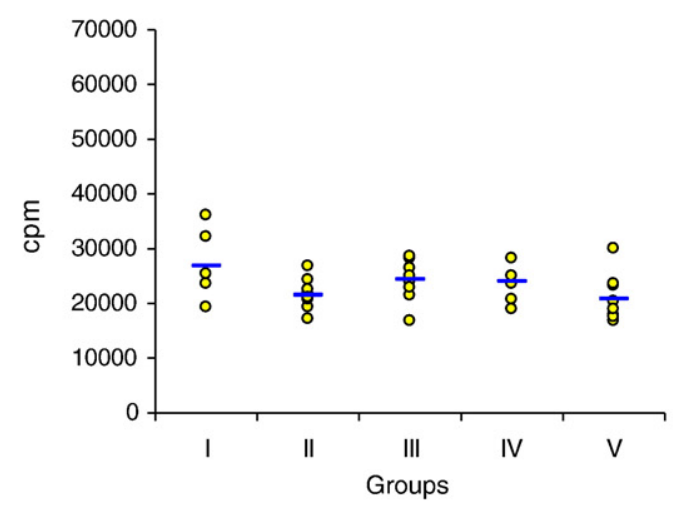


A

concanavalin A $\quad$ a HBsAg + CpG ODN

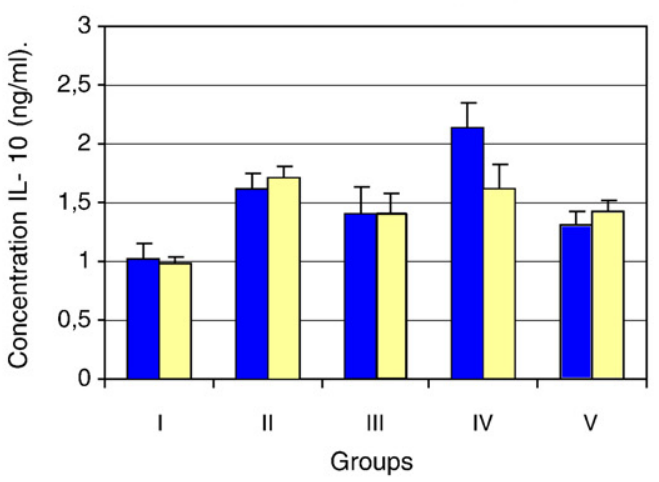

B

(1) Concanavalin A $\square \mathrm{HBsAg}+\mathrm{CpGODN}$

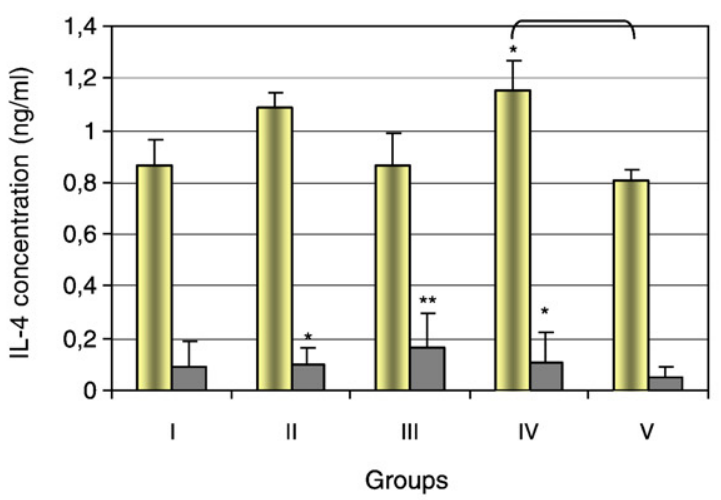

C $\square$ Without stimulus $\boxminus \mathrm{HBsAg}$ 田Con $\mathrm{A} \square \mathrm{HBsAg}+\mathrm{CpG}$ ODN

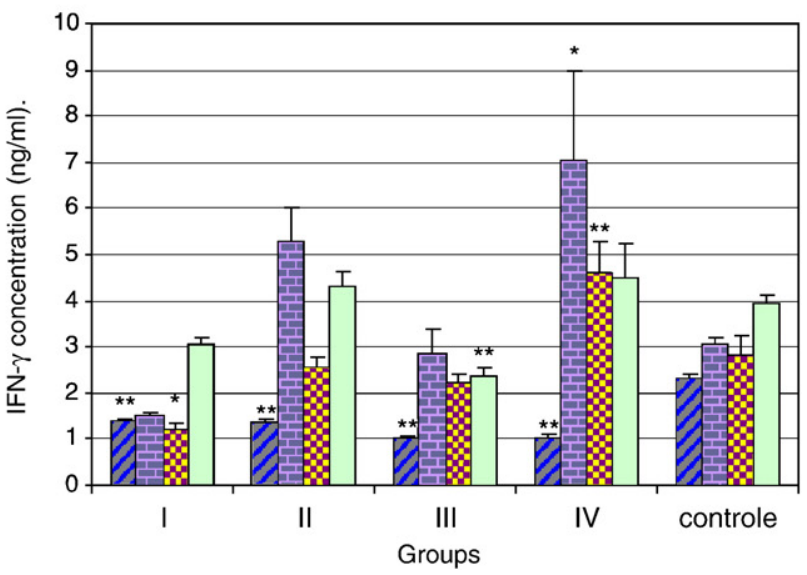

Fig. 3. Cytokine production by splenocytes. The spleens were harvested at 4 week post boost and suspension of individual spleen cells were cultured with either medium alone or in the presence of different stimulus (HBsAg, Con A and HBsAg+CpGODN). Each bar corresponds to the group geometric mean plus the standard error of the mean. Comparisons were made between treatment groups and control (group V). ${ }^{*} p<0.05$ and ${ }^{* *} p<0.01$. A) -Data are IL-10; B) - Data are IL-4; C) -Data are IFN- $\gamma$.

After cell incubation with different compounds, the production of IL-10, IL- 4 and IFN- $\gamma$ cytokines were also analysed in the culture supernatants. It was not possible to detect IL-10 in the supernatants of splenocytes cultured without mitogenic compounds or incubated in the presence of the HBsAg (data not shown). On the other hand, in vitro stimulation with con $A$ (positive control) or with antigen plus the CpG ODN resulted in a production of IL-10 that was similar in almost all the groups (Fig. 3A). To note that splenocytes from group IV, stimulated with Con A seemed to be able to produce a significantly higher amount of IL-10, however the increase found is only 1.6-fold when compared to the control group, therefore, probably of low biological significance.

The profile of the cell-mediated immune response, specifically IFN$\gamma$ (Th1) and IL-4 (Th2) cytokines, was also examined. Groups I and II (vaccinated with the HBsAg associated with coated nanoparticles and with coated nanoparticles + adjuvant, respectively) did not show any modification of the production of IL-4, when compared to the control group (data not shown). Conversely, at the same time point, mice from groups III and IV showed low IL-4 levels (data not shown), in close proximity to undetectable levels. On the other hand, following splenocyte in vitro stimulation with a solution containing $\mathrm{HBsAg}+$ CpG ODN, a small, but significant IL-4 enhancement was observed in almost all the vaccinated groups (Fig. 3B). At the same time, lower amounts of interferon- $\gamma$ in the supernatants of the splenocytes cultured for $96 \mathrm{~h}$ without any mitogen, compared to the non vaccinated mice (control group), were observed in all vaccinated groups (Fig. 3C). However, a different result was obtained when the splenocytes were incubated with different mitogens. In the presence of the HBsAg, only group IV showed a higher concentration $(p<0.05)$ of the interferon, but with a high standard deviation. With regard to the in vitro stimulation with HBsAg plus CpGODN, group III produced a lower amount of the interferon $(p<0.01)$. Also of note is the comparison between the two groups vaccinated with formulations containing the CpG ODN, groups II and III. In group III, a significant $(p<0.01)$ decrease in the concentration of the $\gamma$-IFN in the supernatants of the cells in vitro stimulated with the antigen plus the adjuvant was observed.

The ability of CPG ODN as adjuvant to induce Th1 type cytokines has been previously demonstrated [10]. However, according to the cytokine data obtained in this recent study, a clear Th1 cellular immune response induced by $\mathrm{CpG}$ containing formulations was not observed. At the same time, a Th1/Th2 mixed response was observed in the group vaccinated with a saline solution of the antigen, which has also been reported and attributed to the HBsAg itself [23].

3.4. Humoral immune response following subcutaneous administration of HBsAg associated with nanoparticles

\subsubsection{Systemic immune response}

The association of the HBV antigen (group I) to the alginate coated chitosan nanoparticles induced a strong immune response that was 5.3-fold higher than the mean titer found for group IV, vaccinated with the antigen without any adjuvant (Fig. 4A). In group I, both antiHBsAg IgG1 and IgG2a were detected in the serum, however there was a clear predominance of IgG1 (Fig. 4B). The addition of the $\mathrm{CpG}$ ODN to the formulation used on the group I, which was given to group II, resulted in an increase in anti-HBsAg IgG2a antibody titers and a decrease of anti-HBsAg IgG1 antibody titers. Thus, the main impact was the breaking of the clear predominance of the Th2 immune response for the induction of a mixed Th2/Th1 response (Fig. 4C). Moreover, the mean IgG titers were not different between the groups I and II. Consequently, it can be concluded that the immune response differences between the two groups are only qualitative. Finally, group III was vaccinated with $\mathrm{HBsAg}+\mathrm{CpG}$ ODN adsorbed to the uncoated chitosan nanoparticles. A strong HBsAg specific IgG immune response was observed and was 7.7-fold higher than the mean value found for the control group. Moreover, in a comparison of group I with group III, the values of the anti-HBsAg IgG were not significantly different. However, similar to group I, group III showed a predominance of the IgG1 antibody subtype (Th2 profile immune response). The amount of CpG ODN administered to both groups II and III was equal. In group III, the CpG ODN was associated with the chitosan nanoparticles and might was therefore less available to interact with the immune cells. Therefore in group III, CpG seemed to exert a smaller effect, resulting in the decreased ratio of $\operatorname{IgG} 2 \mathrm{a} / \operatorname{IgG} 1$ of 1.0 in the group II to 0.7 in the group III (Fig. 4C). In fact, both groups (II and 
A
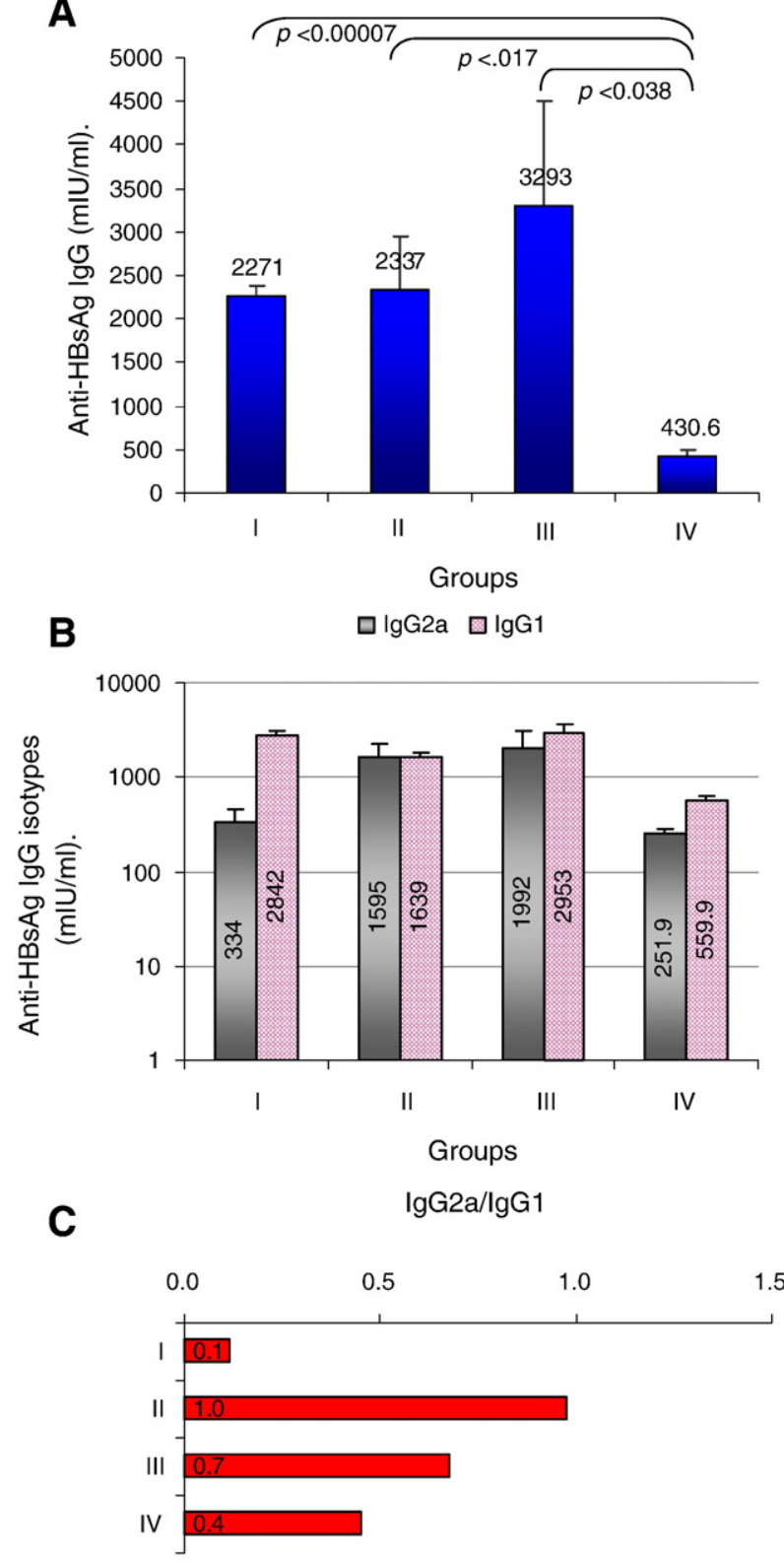

Groups

Fig. 4. A)-Serum anti-HBsAg IgG titers of mice immunized with different formulations of hepatitis B vaccine. Values are expressed as antibody titers of individual mice taken in the end of the experiment. The horizontal bar is the mean of the group. $t$-test was used for analysis of significance between each group and the group IV. B)-Serum anti-HBsAg IgG1 and IgG2a titers (logarithmic scale) of mice immunized with different formulations of hepatitis B vaccine. The bar corresponds to mean titer in each group. Titers were defined as the highest plasma dilution resulting in an absorbance value twice that of nonimmune plasma ( $1 \mathrm{mlU} / \mathrm{ml}=$ mean $+2 \mathrm{SD}$ of the control group). C)-Ratio $\operatorname{lgG} 2 \mathrm{a} /$ IgG1.

III) exhibited similar IgG2a titres, nevertheless the IgG1 titres are different $(t$-test; $p<0.05)$ between the same above groups, being higher in group vaccinated with the formulation where the $\mathrm{CpG}$ was associated with chitosan.

\subsubsection{Mucosal immune response}

In this study, the quantification of anti-HBsAg sIgA was performed on extracts of fresh faeces, collected two days before the end of the experiment. As expected, the subcutaneous administration of the vaccine formulations was unable to induce the production anti-HBsAg IgA (data not shown).

\section{Discussion}

We recently described a new delivery system composed of a chitosan core to which the antigen was adsorbed and which was successively coated with sodium alginate. One advantage of this delivery system is that the antigen is encapsulated under nonstressful conditions, such that the biological properties of the antigen are expected to remain intact. In this study, the potential of chitosan nanoparticles as an adjuvant for the hepatitis B surface antigen administered subcutaneously, was evaluated for the first time. The adjuvant effect of the alginate coated chitosan nanoparticles (group I) was clearly demonstrated by the production of high anti-HBsAg IgG titers with a clear dominance of Th2 type antibodies (IgG1 $>\operatorname{IgG} 2 \mathrm{a})$. A similar behaviour ( $\operatorname{IgG} 1>\operatorname{IgG} 2 \mathrm{a})$ was recently reported with a different type of nanoparticles and antigens, like tetanus toxoidloaded poly(D, L-lactic-co-glycolic acid) nanoparticles [18] or with liposome-encapsulated influenza subunits [24], both administered subcutaneously. Alginate coated chitosan nanoparticles stimulated both Th1 and Th2 type antibodies, however, IgG2a antibodies were induced at a lower percentage, resulting in a decrease of the IgG2a/ IgG1 ratio from 0.4 (control group) to 0.1 . It can be considered that the Th2 immune profile was enhanced, when the alginate coated chitosan nanoparticles were used as an adjuvant. A similar observation was recently reported by us with the same mice strain [25] and after intranasal administration of the antigen associated with alginate coated nanoparticles. Moreover, the same previous study also revealed that the induction of a Th2 immune response is also a characteristic of the hepatitis B commercial formulation (adjuvanted with alum). Likewise, the enhancement of the Th2 profile immune response, after intranasal immunization with a formulation containing chitosan nanoparticles associated to genetically detoxified diphtheria toxin, was reported by others, as well [26].

Analysing the cellular immune responses, it was possible to observe a higher proliferation capability of the spleen cells, stimulated in vitro with Con $\mathrm{A}$, from group vaccinated with HBsAg associated with coated nanoparticles. However, an up-regulation of Th2 type cytokines (i.e., IL-4) produced by the same splenocytes was not observed. Furthermore, a down-regulation of IFN- $\gamma$ may indicate that natural killer T (NKT) and T cells (Th1 type cells), which are both IFN- $\gamma$ producers [13], were less activated in comparison with the same cells from the unvaccinated mouse group.

It has been demonstrated that a cell-mediated immune response, particularly a Th1 response, is important not only for preventing, but also for overcoming HBV infections $[13,27]$ and eliminating the virus from the infected cells [12]. At the same time, it has been suggested that the conventional vaccine is able to elicit a high humoral immune response, but failed to elicit a cell-mediated immune response, rendering it ineffective for the treatment of chronic hepatitis $B$ infection [28]. Therefore, the study of new potential adjuvants with emphasis on their capacity to induce a cell-mediated immune response has been, in the last years, reported in the literature [2830]. In the present study CpG ODN 1826 was included in the formulation (group II) to enhance the level of response or to focus the response through a Th1 pathway $[10,16,17,31]$. In fact an increase in the anti-HBsAg specific IgG2a antibodies and a slight decrease in IgG1 titers were observed in group II, but the total titer of HBsAg specific IgG antibodies was not significantly different from the previous group (Group I). Therefore, in this study, the ability of the CpG ODN to induce a Th1 profile immune response was confirmed once more. On the other hand, an additive effect with the nanoparticles was not observed, most likely because chitosan nanoparticles seemed to be already a strong adjuvant. A similar profile of humoral immune response was recently described with tetanus toxoid-loaded PLGA microspheres and CpG ODN in solution [18]. In contrast, on the same cited study the inclusion of $\mathrm{CPG}$ ODN in nanoparticles with tetanus toxoid resulted in the enhancement of interferon production in all cases. 
Coated chitosan nanoparticles were originally designed for transporting recombinant protein antigens through mucosal surfaces where the presence of proteolytic enzymes or the acid environment of the stomach might destroy the antigen previously adsorbed to the surface of the particles. The coating with alginate of the chitosan nanoparticles might not be necessary when the delivery system is going to be administered by the subcutaneous route. So, in order to test this hypothesis a more simple formulation was studied. In this case, the effect of the co-adsorption of the antigen and the CpG ODN on chitosan nanoparticles was tested, avoiding the coating procedure with sodium alginate. Furthermore, a second advantage of this delivery system is related with its ability to present multiple copies of the antigen on the surface, an effect which has been shown to be optimal for B cell activation [32]. Moreover, the co-adsorption of the antigen and the CpG ODN to the same nanoparticles has been designated as the ideal formulation [33-36]. The s.c. vaccination with this formulation resulted in the highest specific IgG titre and a mixed Th1/Th2 immune response [24]. Interestingly, comparing the IgG2a/ IgG1 ratio of this group with the previous one (group II), where the $\mathrm{CpG}$ was not associated with the nanoparticles, a lower increase of the IgG2a was observed. All the observations seemed to support the idea that the free CpG ODN (not associated with the chitosan nanoparticles) was more efficacious, presumably because it was more accessible to interact with immune cells. By contrast, other studies on particulate delivery of CpG, either surface adsorbed [37], in liposomes [34], or in PLGA nanoparticles [18], found a stronger effect when CpG ODN was encapsulated in the delivery system in comparison with CpG ODN in solution. This feature was considered important since it would allow for a decrease in dosage of $\mathrm{CpG}$ ODN administered while obtaining the same effect. Two different mechanisms can be discussed to explain our results. The first is related to the frequently reported strong affinity between the CpG ODN and the cationic chitosan polymer $[38,39]$, which may cause a slow release of the $\mathrm{CpG}$ in vivo. Therefore, the amount of free $\mathrm{CPG}$ ready to be internalized by the target cells is lower when compared to the situation where the CpG ODN was given in solution. The second putative mechanism is that the internalization of the CpG ODN into the cells is a prerequisite before it can bind to Tolllike receptor 9 (TLR9) present within a number of mouse immune cells and subsequently trigger the immune response [36,40]. As supported by theory and also by some experimental evidence [41], the internalization of the $\mathrm{CPG}$ ODN would be facilitated if it was administered in association with chitosan nanoparticles. However, aggregation of the nanoparticles might occur in vivo compromising the internalisation of the nanoparticles. This hypothesis cannot be totally excluded and will be evaluated in future studies. Finally, even under the assumption that chitosan nanoparticles have been internalized to some extent, the release of the CPG ODN from the particles after their intracellular uptake should also be considered an important issue which influences the efficacy of a vaccine formulation.

Another important advantage of the association of the $\mathrm{CpG}$ with the nanoparticles is related with the protective effect against enzymatic degradation of the CPG ODN. This aspect is particularly important in the enzyme rich intestinal mucosa as previously reported [41-43]. So, due to their unique and interesting properties, which have been reviewed recently in several papers [3,44-47], chitosan and chitosan nanoparticles have been used in mucosal delivery systems for vaccination using several antigens and mucosal routes [26,48-51]. However, their potential as adjuvants for parenteral vaccination has been less studied. In a very recent study [52], a chitosan solution was explored as an adjuvant for subcutaneous vaccination of mice with a model antigen. It was found that chitosan enhanced the antigenspecific antibody titers over five-fold and antigen-specific CD4+ lymphocytes proliferation over six-fold. Mechanistic studies performed by the same authors revealed that the antigen depot and a transient cellular expansion in draining lymph nodes induced by chitosan may explain its adjuvant properties [52].

\section{Conclusion}

For the first time, the adjuvant effect of alginate coated chitosan nanoparticles for the hepatitis B surface antigen was evaluated after subcutaneous application in mice. A potent enhancement of the humoral immune response was observed with predominance of Th2 type antibodies. The Th profile immune response was re-directed to Th1 type when the antigen-loaded chitosan nanoparticles were codelivered with the CpG ODN 1826 in solution. A third formulation, in which the antigen and the adjuvant were both adsorbed to chitosan nanoparticles was also evaluated. No additional important benefits were observed with this formulation. However, the manufacturing simplicity of this last formulation makes it a potential basis for future formulation improvements. All chitosan-based formulations, containing CpGODN have shown a great potential for the improvement of the currently licensed HBV vaccines, particularly making them useful for the treatment of chronic hepatitis B, where Th-1 cellular immune response induction is required. Further doseresponse studies are required in order to evaluate and compare with the existing vaccines in the market and with other adjuvants in clinical investigation.

\section{Acknowledgements}

The authors wish to thank Dr. Sandra Giannini and Dr. Martine Wettendorf (GlaxoSmithKline, Biologicals, Belgium) for providing the hepatitis B vaccine. The authors also acknowledge Dr. Sandra Giannini and Dr. Ulrike Krause (GSK) for their valuable comments to this manuscript.

The work was in part supported by FCT and Programa Operacional Ciência e Inovação 2010 (POCI2010) and Feder in the Project POCI/ CVT/59840/2004.

\section{References}

[1] Porporatto C, Bianco ID, Correa SG. Local and systemic activity of the polysaccharide chitosan at lymphoid tissues after oral administration. J Leukoc Biol 2005;78(1):62-9.

[2] Babensee JE, Paranjpe A. Differential levels of dendritic cell maturation on different biomaterials used in combination products. J Biomed Mater Res A 2005;74(4):503-10.

[3] Chopra S, Mahdi S, Kaur J, Iqbal Z, Talegaonkar S, Ahmad FJ. Advances and potential applications of chitosan derivatives as mucoadhesive biomaterials in modern drug delivery. J Pharm Pharmacol 2006;58(8):1021-32.

[4] Thanou M, Verhoef JC, Junginger HE. Chitosan and its derivatives as intestinal absorption enhancers. Adv Drug Deliv Rev 2001;50(Suppl 1):S91-101.

[5] van der Lubben IM, Verhoef JC, Borchard G, Junginger HE. Chitosan for mucosal vaccination. Adv Drug Deliv Rev 2001:52(2):139-44.

[6] O'Hagan D. Microparticles as vaccine delivery systems. In: Schijins V, O'Hagan D, editors. Immunopotentiators in modern vaccines. 1st ed. Academic Press; 2006. p. 123-47.

[7] Craven DE, Awdeh ZL, Kunches LM, Yunis EJ, Dienstag JL, Werner BG, et al. Nonresponsiveness to hepatitis B vaccine in health care workers. Results of revaccination and genetic typings. Ann Intern Med 1986;105(3):356-60.

[8] Mast EE, Weinbaum CM, Fiore AE, Alter MJ, Bell BP, Finelli L, et al. A comprehensive immunization strategy to eliminate transmission of hepatitis $B$ virus infection in the United States: recommendations of the Advisory Committee on Immunization Practices (ACIP) Part II: immunization of adults. MMWR Recomm Rep 2006;55(RR-16):1-33 quiz CE1-4.

[9] Rahman F, Dahmen A, Herzog-Hauff S, Bocher WO, Galle PR, Lohr HF. Cellular and humoral immune responses induced by intradermal or intramuscular vaccination with the major hepatitis B surface antigen. Hepatology 2000;31(2):521-7.

[10] Weeratna RD, Brazolot Millan CL, McCluskie MJ, Davis HL. CpG ODN can re-direct the Th bias of established Th2 immune responses in adult and young mice. FEMS Immunol Med Microbiol 2001;32(1):65-71.

[11] Steinke JW, Borish L. 3. Cytokines and chemokines. J Allergy Clin Immunol 2006;117(2 Suppl Mini-Primer):S441-5.

[12] Constant SL, Bottomly K. Induction of Th1 and Th2 CD4+ T cell responses: the alternative approaches. Annu Rev Immunol 1997;15:297-322.

[13] Rehermann B, Nascimbeni M. Immunology of hepatitis B virus and hepatitis C virus infection. Nat Rev Immunol 2005;5(3):215-29.

[14] Moldoveanu Z, Love-Homan L, Huang WQ, Krieg AM. CpG DNA, a novel immune enhancer for systemic and mucosal immunization with influenza virus. Vaccine 1998;16(11-12):1216-24

[15] McCluskie MJ, Weeratna RD, Payette PJ, Davis HL. Parenteral and mucosal primeboost immunization strategies in mice with hepatitis B surface antigen and CpG DNA. FEMS Immunol Med Microbiol 2002;32(3):179-85. 
[16] Osorio JE, Zuleger CL, Burger M, Chu Q, Payne LG, Chen D. Immune responses to hepatitis B surface antigen following epidermal powder immunization. Immunol Cell Biol 2003;81(1):52-8

[17] Davis HL, Weeratna R, Waldschmidt TJ, Tygrett L, Schorr J, Krieg AM. CpG DNA is a potent enhancer of specific immunity in mice immunized with recombinant hepatitis B surface antigen. J Immunol 1998;160(2):870-6.

[18] Diwan M, Tafaghodi M, Samuel J. Enhancement of immune responses by codelivery of a $\mathrm{CpG}$ oligodeoxynucleotide and tetanus toxoid in biodegradable nanospheres. J Control Release 2002;85(1-3):247-62.

[19] Borges O, Borchard G, Verhoef JC, de Sousa A, Junginger HE. Preparation of coated nanoparticles for a new mucosal vaccine delivery system. Int J Pharm 2005;299(1-2):155-66.

[20] Borges O, Cordeiro-da-Silva A, Romeijn SG, Amidi M, Sousa AD, Borchard G, et al. Uptake studies in rat Peyer's patches, cytotoxicity and release studies of alginate coated chitosan nanoparticles for mucosal vaccination. J Control Release 2006;114 (3):348-58

[21] Borges O, Tavares J, de Sousa A, Borchard G, Junginger HE, Cordeiro-da-Silva A. Evaluation of the immune response following a short oral vaccination schedule with hepatitis B antigen encapsulated into alginate-coated chitosan nanoparticles. Eur J Pharm Sci 2007;32(4-5):278-90.

[22] Cordeiro-da-Silva A, Tavares J, Araujo N, Cerqueira F, Tomas A, Kong Thoo Lin P, et al. Immunological alterations induced by polyamine derivatives on murine splenocytes and human mononuclear cells. Int Immunopharmacol 2004;4(4):547-56

[23] Weeratna RD, McCluskie MJ, Xu Y, Davis HL. CpG DNA induces stronger immune responses with less toxicity than other adjuvants. Vaccine 2000;18(17):1755-62.

[24] Joseph A, Louria-Hayon I, Plis-Finarov A, Zeira E, Zakay-Rones Z, Raz E, et al. Immune response by nasal delivery of hepatitis $B$ surface antigen and codelivery of a CPG ODN in alginate coated chitosan nanoparticles. Eur J Pharm Biopharm 2008;69(2):405-16.

[25] Borges O, Cordeiro-da-Silva A, Tavares JNS, de Sousa A, Borchard G, Junginger HE. Immune response by nasal delivery of hepatitis B surface antigen and codelivery of a CPG ODN in alginate coated chitosan nanoparticles. Eur J Pharm Biopharm 2008;69(2):405-16.

[26] McNeela EA, O'Connor D, Jabbal-Gill I, Illum L, Davis SS, Pizza M, et al. A mucosal vaccine against diphtheria: formulation of cross reacting material (CRM(197)) of diphtheria toxin with chitosan enhances local and systemic antibody and Th2 responses following nasal delivery. Vaccine 2000;19(9-10):1188-98.

[27] Jung MC, Pape GR. Immunology of hepatitis B infection. Lancet Infect Dis 2002;2 (1):43-50.

[28] Goyal AK, Rawat A, Mahor S, Gupta PN, Khatri K, Vyas SP. Nanodecoy system: a novel approach to design hepatitis B vaccine for immunopotentiation. Int J Pharm 2006;309(1-2):227-33.

[29] He X, Jiang L, Wang F, Xiao Z, Li J, Liu LS, et al. Augmented humoral and cellular immune responses to hepatitis B DNA vaccine adsorbed onto cationic microparticles. J Control Release 2005;107(2):357-72.

[30] Chong CS, Cao M, Wong WW, Fischer KP, Addison WR, Kwon GS, et al. Enhancement of $\mathrm{T}$ helper type 1 immune responses against hepatitis $\mathrm{B}$ virus core antigen by PLGA nanoparticle vaccine delivery. J Control Release 2005;102 (1):85-99.

[31] Manning BM, Enioutina EY, Visic DM, Knudson AD, Daynes RA. CpG DNA functions as an effective adjuvant for the induction of immune responses in aged mice. Exp Gerontol 2001;37(1):107-26.

[32] Bachmann MF, Rohrer UH, Kundig TM, Burki K, Hengartner H, Zinkernagel RM. The influence of antigen organization on B cell responsiveness. Science 1993;262 (5138):1448-51.
[33] Gursel I, Gursel M, Ishii KJ, Klinman DM. Sterically stabilized cationic liposomes improve the uptake and immunostimulatory activity of $\mathrm{CpG}$ oligonucleotides. J Immunol 2001;167(6):3324-8.

[34] Gursel M, Tunca S, Ozkan M, Ozcengiz G, Alaeddinoglu G. Immunoadjuvant action of plasmid DNA in liposomes. Vaccine 1999;17(11-12):1376-83.

[35] Li WM, Bally MB, Schutze-Redelmeier MP. Enhanced immune response to T-independent antigen by using $\mathrm{CpG}$ oligodeoxynucleotides encapsulated in liposomes. Vaccine 2001;20(1-2):148-57.

[36] Klinman DM, Currie D, Gursel I, Verthelyi D. Use of CpG oligodeoxynucleotides as immune adjuvants. Immunol Rev 2004;199:201-16.

[37] Singh M, Ott G, Kazzaz J, Ugozzoli M, Briones M, Donnelly J, et al. Cationic microparticles are an effective delivery system for immune stimulatory cpG DNA. Pharm Res 2001;18(10):1476-9.

[38] Danielsen S, Varum KM, Stokke BT. Structural analysis of chitosan mediated DNA condensation by AFM: influence of chitosan molecular parameters. Biomacromolecules 2004;5(3):928-36.

[39] Dang JM, Leong KW. Natural polymers for gene delivery and tissue engineering. Adv Drug Deliv Rev 2006;58(4):487-99.

[40] Krieg AM. Therapeutic potential of Toll-like receptor 9 activation. Nat Rev Drug Discov 2006;5(6):471-84.

[41] Wu KY, Wu M, Fu ML, Li H, Yang Y, Zhang H, et al. A novel chitosan CpG nanoparticle regulates cellular and humoral immunity of mice. Biomed Environ Sci 2006;19(2):87-95.

[42] Fu ML, Ying SC, Wu M, Li H, Wu KY, Yang Y, et al. Regulating effects of novel CpC chitosan-nanoparticles on immune responses of mice to porcine paratyphoid vaccines. Biomed Environ Sci 2006;19(4):315-22.

[43] Richardson SC, Kolbe HV, Duncan R. Potential of low molecular mass chitosan as a DNA delivery system: biocompatibility, body distribution and ability to complex and protect DNA. Int J Pharm 1999;178(2):231-43.

[44] Davis SS. The use of soluble polymers and polymer microparticles to provide improved vaccine responses after parenteral and mucosal delivery. Vaccine 2006;24(Suppl 2) S2-7-10.

[45] Prego C, Torres D, Alonso MJ. The potential of chitosan for the oral administration of peptides. Expert Opin Drug Deliv 2005;2(5):843-54.

[46] Prabaharan M, Mano JF. Chitosan-based particles as controlled drug delivery systems. Drug Deliv 2005;12(1):41-57.

[47] Kurita K. Chitin and chitosan: functional biopolymers from marine crustaceans. Mar Biotechnol (NY) 2006;8(3):203-26.

[48] van der Lubben IM, Kersten G, Fretz MM, Beuvery C, Coos Verhoef J, Junginger HE. Chitosan microparticles for mucosal vaccination against diphtheria: oral and nasal efficacy studies in mice. Vaccine 2003;21(13-14):1400-8.

[49] Baudner BC, Verhoef JC, Giuliani MM, Peppoloni S, Rappuoli R, Del Giudice G, et al Protective immune responses to meningococcal $\mathrm{C}$ conjugate vaccine after intranasal immunization of mice with the LTK63 mutant plus chitosan or trimethyl chitosan chloride as novel delivery platform. J Drug Target 2005;13(8-9):489-98.

[50] Bivas-Benita M, van Meijgaarden KE, Franken KL, Junginger HE, Borchard G Ottenhoff $\mathrm{TH}$, et al. Pulmonary delivery of chitosan-DNA nanoparticles enhances the immunogenicity of a DNA vaccine encoding HLA-A*0201-restricted T-cell epitopes of Mycobacterium tuberculosis. Vaccine 2004;22(13-14):1609-15.

[51] Kang ML, Kang SG, Jiang HL, Shin SW, Lee DY, Ahn JM, et al. In vivo induction of mucosal immune responses by intranasal administration of chitosan microspheres containing Bordetella bronchiseptica DNT. Eur J Pharm Biopharm 2006;63(2):215-20.

[52] Zaharoff DA, Rogers CJ, Hance KW, Schlom J, Greiner JW. Chitosan solution enhances both humoral and cell-mediated immune responses to subcutaneous vaccination. Vaccine 2007;25(11):2085-94. 